\title{
INVARIANT LINEAR FUNCTIONALS
}

\author{
W. RANDOLPH WOODWARD* and R. R. CHIVUKULA
}

(Received 9 June 1970)

Communicated by J. B. Miller

\section{Introduction}

Let $B$ be a Banach space and let $\mathscr{L}(B)$ denote the space of all bounded inear operators from $B$ to $B$, which is a Banach algebra under composition of operators as multiplication. By a semigroup of operators $G$ on $B$, we mean a norm bounded subset $G$ of $\mathscr{L}(B)$ which is a subsemigroup in the multiplicative structure of $\mathscr{L}(B)$. The purpose of this paper is to study the existence of nonzero continuous linear functionals on $B$ invariant under $G$, that is given $B$ and $G$, does there exist $\mu \in B^{*}$, with $\mu \neq 0$, such that $\mu(S x)=\mu(x)$ for all $x \in B$ and $S \in G$. This question is an attempt to generalize the familiar concepts of invariant means and amenability of semigroups. If $H$ is any semigroup and $m(H)$ is the Banach space of all bounded real valued functions on $H$ with supremum norm, then a mean is a positive normalized continuous linear functional on $m(H)$. A mean is called (left) [right] invariant if it is invariant under (left) [right] translations and $H$ is called (left) [right] amenable if there exists such a mean; e.g., $F$ is a left invariant mean if $F \in m(H)^{*}$ is such that (i) $\|F\|=1$, (ii) $F(x) \geqq 0$ if $x(g) \geqq 0$ for all $g \in H$, (iii) $F\left(x_{g}\right)=F(x)$ for all $x \in m(H)$ and $g \in G$ where $x_{g}(h)=x(g h)$ for all $h \in H$. Amenability of semigroups has been studied extensively in recent years, for example see Day [2] or Hewitt and Ross [6] for an introduction, and Day [3] for a comprehensive survey. If $H$ is a (locally compact) topological group it is appropriate to replace $m(H)$ by one of the following: $C(H) \equiv$ all bounded continuous functions; $L U C(H) \equiv$ all functions bounded and left uniformly continuous; similarly $R U C(H)$ and $U C(H)$; finally $L^{\infty}(H) \equiv$ all essentially bounded measurable (with respect to Haar measure) functions with essential supremum norm. See Greenleaf [5] for this topological case. It is obvious that if $B$ is chosen to be one of these Banach spaces and $G$ to be the set of operators induced on $B$ by the naturally available translations (left or right)

* For the most part, the results in this paper are in a disseration submitted in partial fulfill. ment of the requirements for a Ph.D. degree at the University of Nebraska, Lincoln, Nebraska. 
of $H$, then $G$ is a semigroup of operators on $B$ and amenability of $H$ is a special case of the question we posed at the beginning. Our concept of a linear functional invariant under a semigroup of operators also includes several other instances where a Banach space of functions is chosen and a semigroup of operators is then specified; usually these operators are induced by transformations on the domain space of the functions. We give two such instances: (1) let $X$ be a compact Hausdorff space, $C(X)$ continuous functions on $X$ and $\mathscr{T}$ be a semigroup of homeomorphisms of $X$. See Rich [8] for some results in this setting where he is seeking a measure on $X$ invariant under $\mathscr{T}$. For each $\tau \in \mathscr{T}$, if we define $S_{\tau}: C(X) \rightarrow C(X)$ by

$$
\left(S_{\tau} f\right)(x)=f(\tau x),
$$

then it is obvious that $G=\left\{S_{\tau}: \tau \in \mathscr{T}\right\}$ is an operator semigroup on $C(X)$ and a linear functional invariant under $G$ is a measure invariant under $\mathscr{T}$ (via Rieszrepresentation Theorem). (2) Next following Wilde and Tayachandran [9], let $X$ be an arbitrary set and $\mathscr{T}$ a semigroup of mappings of $X$ into $X$. Each $\tau \in \mathscr{T}$ induces a bounded linear operator $S_{\tau}$ on $m(X)$; let

$$
G=\left\{S_{\tau}: \tau \in \mathscr{T}\right\} .
$$

Call the pair $(X, \mathscr{T})$ a transformation semigroup and say that it is amenable if there is a $G$-invariant mean on $m(X)$. It is obvious that $G$ is an operator semigroup and amenability of $(X, \mathscr{T})$ is a particular case of our question.

Thus it is clear that the concept of a linear functional invariant under a semigroup of operators is very general. In this paper we study the existence of such functionals. We let $B$ be a Banach space and $G$ a semigroup of operators on $B$. If $\mu \in B^{*}$, the normed conjugate of $B$, and $\mu(S x)=\mu(x)$ for all $x \in B$ and $S \in G$, then $\mu$ is called $G$-invariant. If such a nonzero $\mu$ exists we say $B$ is $G$-amenable. Since $G \subseteq \mathscr{L}(B)$ and this latter space has several topologies available, we choose to consider $G$ as a topological semigroup. As such it is appropriate to consider the amenability of $G$ in the sense of existence of invariant an mean on $C(G)$. For a fixed $x \in B$ let $K(x)$ denote the weakly closed convex hull of the orbit of $x$, $\{S x: S \in G\}$. An example of our results is;

THEOREM. If $G$ is right amenable and if there exists $x_{0} \in B$ such that $0 \notin K\left(x_{0}\right)$, then $B$ is $G$-amenable. Conversely if $B$ is $G$-amenable then there exists $x_{0} \in B$ such that $0 \notin K\left(x_{0}\right)$.

Section 2 contains a brief discussion of semigroups of operators and we develop a mapping

$$
\phi_{\xi}: B \rightarrow C(G)
$$

which transforms the operators in $G$ into right translations on $C(G)$. Besides being our main tool in the discussion of $G$-amenability presented in Section 3, 
this mapping is of some interest in itself and we have included some results. See Theorems 1, 2 and Corollary 2. We note that our results do not yield any significantly new results in the theory of invariant means. On the contrary, our aim has been to utilize the available theory of amenable semigroups to obtain $G$-invariant linear functionals. $(G$, s.o.) is a topological semigroup with separately continuos multiplication.

2.

Let $G$ be a norm-bounded (algebraic) semigroup in $\mathscr{L}(B)$. We write $(G$, w.o.), $(G$, s.o.) and $(G$, u.) respectively to mean that $G$ is considered topologized under the relative weak operator, strong operator and uniform topologies of $\mathscr{L}(B)$.

LEMMA 1. (a) $(G, \mathrm{u}$.) and $(G$, s.o.) are topological semigroups with jointly continuous multiplication. (G,w.o.) is a topological semigroup with separately continuous multiplication.

(b) Let $G$ be a semigroup in $\mathscr{L}(B)$ and $\bar{G}$ its s.o. closure. Then $(\bar{G}$, w.o.) is a topological semigroup.

Proof. (a) Straightforward and hence omitted. (b) By part (a), to show that $(\bar{G}$, w.o. $)$ is a topological semigroup, it is sufficient to show that $\bar{G}$ is a bounded semigroup. Since $G$ is bounded (by $N>0$ ), so is $\bar{G}$. Let $\varepsilon>0, x$ in $B$ and $S, T$ in $\bar{G}$; then there are nets $\left\{S_{\alpha}\right\}$ and $\left\{T_{\beta}\right\}$ in $G$ such that $S_{\alpha} \rightarrow S$ [s.o.] and $T_{B} \rightarrow T$ [s.o.] Find $\alpha_{0}$ and $\beta_{0}$ such that

and

$$
\left\|S_{\alpha}(T x)-S(T x)\right\|<\frac{\varepsilon}{2} \quad \text { for } \alpha>\alpha_{0}
$$

$$
\left\|T_{\beta} x-T x\right\|<\frac{\varepsilon}{2 N} \quad \text { for } \beta>\beta_{0} \text {. }
$$

Then

$$
\left\|S_{\alpha} T_{b} x-S T x\right\| \leqq\left\|S_{\alpha}\left(T_{B} x-T x\right)\right\|+\left\|S_{\alpha}(T x)-S(T x)\right\|
$$

Thus $S T \in \bar{G}$.

$$
\leqq N\left\|T_{\beta} x-T x\right\|+\frac{\varepsilon}{2}<\varepsilon \quad \text { for } \alpha>\alpha_{0} \text { and } \beta>\beta_{0} .
$$

We adopt some of the notation of [7]. Thus if $\Sigma$ is any semigroup and $X$ a space of functions on $\Sigma$ and $\sigma \in \Sigma$ and $\left(e_{\sigma}\right)\left[r_{\sigma}\right]$ denotes respectively the (left) [right] translation operators then $X$ is called (left) [right] invariant if $x \in X$ implies all $\left(l_{\sigma} x\right)\left[r_{\sigma} x\right]$ are in $X$. If $\Sigma$ is a topological semigroup, $C(\Sigma)$ will denote the Banach space of all continuous bounded real functions under sup norm. An $f \in C(\Sigma)$ is called left uniformly continuous (l.u.c.) if the map $\sigma \rightarrow r_{\sigma} f$ is continuous in the norm. Right uniformly continuous (r.u.c.) functions are defined similarly and $L U C(\Sigma), R U C(\Sigma), U C(\Sigma)$ respectively denote the Banach space of all 1.u.c., 
r.u.c. and u.c. functions on $\Sigma$. Again as in [7], we use the same symbols $l_{\sigma}, r_{\sigma}$ on any invariant linear $X$. Further let

$$
R_{\Sigma}=\left\{r_{\sigma}: \sigma \in \Sigma\right\}
$$

and similarly for $L_{\Sigma}$. Now for any right invariant $X, R_{\Sigma} \subseteq \mathscr{L}(X)$. Thus $R_{\Sigma}$, a semigroup homomorphic to $\Sigma$, can be considered as contained in $\mathscr{L}(C(\Sigma))$ or $\mathscr{L}(U C(\Sigma))$. With either choice, by Lemma $1, R_{\Sigma}$ is a topological semigroup with three possible topologies. However, it matters very much where $R_{\Sigma}$ is and what topology is on it. For example let $H$ denote the usual topological group of real numbers. As a consequence of Theorem 2 of [7] we see that if $R_{H} \subseteq \mathscr{L}(C(H)$, then $\left(R_{H}\right.$; s.o.) is discrete. But if $R_{H} \subseteq \mathscr{L}(U C(H))$ then $\left(R_{H}\right.$; s.o.) is topologically isomorphic with $H$. We now return to the general case of an arbitrary Banach space $B$ and a semigroup of operators $G \subseteq \mathscr{L}(B)$.

Let $\left(G\right.$, w.o.) be a topological semigroup in $\mathscr{L}(B)$ and let $\xi \in B^{*}$. Define $\phi_{\xi}: B \rightarrow C(G)$ by

$$
\left(\phi_{\xi} x\right)(S)=\xi(S x)
$$

for all $x \in B$ and $S \in G$. It is obvious that $\phi_{\xi} x$ is a bounded linear real valued function. The following lemma justifies the use of $C(G)$ as the image space.

Lemma 2. (a) For each $\xi \in B^{*}$ and $x \in B, \phi_{\xi} x \in C(G)$.

(b) For each $S \in G, \phi_{\xi}(S x)=r_{s}\left(\phi_{\xi} x\right)$.

That is each of the maps $\phi_{\xi}$ transforms the operators $S$ into right translations $r_{s}$ on $C(G)$.

(c) If $G$ is $\left(G\right.$, s.o.), then $\phi_{\xi}: B \rightarrow L U C(G)$.

(d) If $G$ is $(G, u)$, then $\phi_{\xi}: B \rightarrow U C(G)$.

Proof. (a) Clearly $\phi_{\xi}$ is linear and bounded in norm by $\|\xi\|$. Let

$$
K=\sup \{\|S\|: S \in G\} \text {. }
$$

To show $\phi_{\xi} x \in C(G)$, let $S_{\alpha} \rightarrow S$. Then

$$
\left(\phi_{\xi} x\right)\left(S_{a}\right)=\xi\left(S_{\sigma} x\right) \rightarrow \xi(S x)=\left(\phi_{\xi} x\right)(S)
$$

by definition of [w.o.] convergence.

(b) For $T \in G$,

$$
\left(\phi_{\xi} S x\right)(T)=\xi(T S x)=\left(\phi_{\xi} x\right)(T S)=\left(r_{s} \phi_{\xi} x\right)(T) .
$$

(c) Let $S_{\alpha} \rightarrow S$ in $\left(G\right.$, s.o.) and $x \in B$. Find $\alpha_{0}$ such that $\alpha>x_{0}$ implies

$$
\left\|S_{\alpha} x-S x\right\|<\frac{\varepsilon}{K\|\xi\|} .
$$

Then 


$$
\begin{aligned}
\left\|r_{s_{x}} \phi_{\xi} x-r_{s} \phi_{\xi} x \mid\right\| & \left.=\sup _{T}\left\{\mid r_{s_{\alpha}} \phi_{\xi} x-r_{s} \phi_{\xi} x\right)(T) \mid\right\} \\
& =\sup _{T}\left|\left(T^{*} \xi\right)\left(S_{\alpha} x-S x\right)\right| \leqq \sup _{T} K\|\xi\| \cdot\left\|S_{\alpha} x-S x\right\| \\
& <\varepsilon .
\end{aligned}
$$

Thus $r_{s_{\alpha}} \phi_{\xi} x \rightarrow r_{s} \phi_{\xi} x$ in $C(G)$ and $\phi_{\xi} x \in L U C(G)$.

(d) is proved similarly.

A natural question to ask is when will the map $\phi_{\xi}$ be an isometry; the next theorem gives necessary and sufficient conditions. Let $-G=\{-S: S \in G\}$.

THEOREM 1. The map $\phi_{\xi}$ will be an isometry if and only if $K$, the $w^{*}$-closed convex hull of

$$
\left\{S^{*} \xi: S \in G \cup-G\right\}
$$

is equal to the unit ball $U_{0}$ of $B^{*}$.

Proof. We will use the following variation of the Krein-Mil'man Theorem, Day [1]: $K=U_{0}$ if and only if for each $f \in\left(B^{*}, w^{*}\right)^{*}$,

$$
\sup \{f(\eta): \eta \in K\}=\sup \left\{f(\eta): \eta \in U_{0}\right\}
$$

Assume $\phi_{\xi}$ is an isometry and $f \in\left(B^{*}, w^{*}\right)^{*}$. Then there is an $x_{0} \in B$ such that $f=Q x_{0}$ where $Q$ is the natural map of $B$ to $B^{* *}$. Then

$$
\begin{aligned}
\sup \{f(\eta): \eta \in K\} & =\sup \left\{\left(Q x_{0}\right)(\eta): \eta \in K\right\} \\
& =\sup \left\{\left(Q x_{0}\right)\left(S^{*} \xi\right): S \in G \cup-G\right\} \\
& =\sup \left\{\left(S^{*} \xi\right)\left(x_{0}\right): S \in G \cup-G\right\} \\
& =\sup \left\{\xi\left(S x_{0}\right): S \in G \cup-G\right\} \\
& =\sup \left\{\left(\phi_{\xi} x_{0}\right)(S): S \in G \cup-G\right\} \\
& =\sup \left\{\left|\left(\phi_{\xi} x_{0}\right)(S)\right|: S \in G\right\}=\left\|\phi_{\xi} x_{0}\right\| \\
& =\left\|x_{0}\right\|=\sup \left\{\eta\left(x_{0}\right): \eta \in U_{0}\right\} \\
& =\sup \left\{\left(Q x_{0}\right)(\eta): \eta \in U_{0}\right\} \\
& =\sup \left\{f(\eta): \eta \in U_{0}\right\} .
\end{aligned}
$$

Thus $K=U_{0}$.

Assume now that $K=U_{0}$. Then for $x_{0} \in B$ we have

$$
\begin{aligned}
\left\|\phi_{\xi} x_{0}\right\| & =\sup \left\{\left|\phi_{\xi} x_{0}(S)\right|: S \in G\right\}=\sup \left\{\left|\xi\left(S x_{0}\right)\right|: S \in G\right\} \\
& =\sup \left\{\xi\left(S x_{0}\right): S \in G \cup-G\right\}=\sup \left\{\left(S^{*} \xi\right)\left(x_{0}\right): S \in G \cup-G\right\} \\
& =\sup \left\{\left(Q x_{0}\right)\left(S^{*} \xi\right): S \in G \cup-G\right\}=\sup \left\{\left(Q x_{0}\right)(\eta): \eta \in K\right\}
\end{aligned}
$$




$$
\begin{aligned}
\sup \left\{\left(Q x_{0}\right)(\eta): \eta \in U_{0}\right\} & =\sup \left\{\eta\left(x_{0}\right): \eta \in U_{0}\right\} \\
& =\left\|x_{0}\right\| .
\end{aligned}
$$

Thus $\phi_{\xi}$ is an isometry.

It is well known that every Banach space $B$ is isometrically isomorphic to a subspace of $C(A)$ where $A$ can be taken to be a compact Hausdorff space. We give the following variation as an application of Theorem 1 .

THEOREM 2. Every Banach space $B$ is isometrically isomorphic to a subspace of $C(G)$ where $G$ is a (closed) topological subsemigroup (of $(\mathscr{L}(B)$, w.o.)).

Proof. Let $B$ be any Banach space and $\xi \in B^{*}$ with $\|\xi\|=1$. Take

$$
G=\{S \in \mathscr{L}(B):\|S\| \leqq 1\}
$$

Then $G$ is a (weak operator) topological semigroup and the closed convex hull of $\left\{S^{*} \xi: S \in G\right\}$ equals the unit ball of $B^{*}$. By Theorem 1, the map $\phi_{\xi}$ is an isometric isomorphism.

It is interesting to note that the condition given in Theorem 1 is independent of the topology chosen for $G$. However, Lemma 2 implies $G$ must have a topology at least as strong as the [w.o.] topology. With this in mind we have

THEOREM 2'. Every Banach space $B$ is isometrically isomorphic to a subspace of $U C(G)$ where $G$ can be taken to be a (closed metrizable topological semigroup (in $\mathscr{L}(B)$ ).

Proof. As in the proof of Theorem $1, \phi_{\xi}$ is an isometry if

$$
G=\{S \in \mathscr{L}(B):\|S\| \leqq 1\}
$$

If $G$ is $(G, u)$, then by Lemma $2(\mathrm{~d}) \phi_{\xi}: B \rightarrow U C(G)$ and $B$ is isometrically isomorphic to a subspace of $U C(G)$.

Contrary to first appearance, Theorem 2' may not be stronger than Theorem 2. This is because the size of $C(G)$ may be increased when the topology of $G$ is increased from [w.o.] to norm.

We note that if $B$ is reflexive then the unit ball of $\mathscr{L}(B)$ is compact [4] in the [w.o.] topology. Thus

Corollary 1. If $B$ is reflexive, then $B$ is isometrically isomorphic to $a$ subspace of $C(G)$ where $G$ is a compact topological semigroup.

3.

In this section we study the following question: Given a Banach space $B$, what conditions on a bounded semigroup $G \subseteq \mathscr{L}(B)$ imply the existence of $\mu \neq 0$ in $B^{*}$ such that $\mu(S x)=\mu(x)$ for all $x \in B$ and $S \in G$ ? If such a $\mu$ exists 
for a given $G$ then $B$ is called $G$-amenable and $\mu$ is called $G$-invariant. We will assume, as we may without loss of generality, that the identity operator $I$ is in $G$.

A natural condition to impose on $G$ is that it be amenable (in the sense of Day [2]). However, this is not sufficient as we can easily see by the example $G=\{I,-I\} . G$ is finite and thus amenable but $B$ is not $G$-amenable since if it were, then there would be a nonzero $\mu$ in $B^{*}$ such that $\mu(I x)=\mu(-I x)$ for all $x \in B$, an impossibility. In fact if $-I$ is in any $G$ (with $I$ in $G$ by assumption), then $B$ is not $G$-amenable. One condition on $G$ which insures that $-I$ and $I$ be not both in $G$ is that $G$ has a nonzero fixed point, that is there is a nonzero $x_{0}$ in $B$ such that $S x_{0}=x_{0}$ for all $S \in G$. This condition is satisfied by the semigroups $R_{\Sigma}$ snd $L_{\Sigma}$ of operators on $m(\Sigma)$ since the function $e(\sigma) \equiv 1$ is fixed under all $r_{\sigma}$ and $l_{\sigma}$.

We will actually show that a condition weaker than this fixed point condition is sufficient.

LEMMA 3. Let $G$ be a bounded semigroup in $\mathscr{L}(B)$. Suppose there is $a \mu \neq 0$ in $C\left(G^{*}\right)$ which is right invariant and suppose there is $a \xi \in B^{*}$ and $x_{0} \in B$ such that $\mu\left(\phi_{\xi} x_{0}\right) \neq 0$. Then $B$ is G-amenable.

PROof. For $\xi$ and $G$ as in Lemma 3 we have $\phi_{\xi}: B \rightarrow C(G)$ by Lemma 2 and thus

$$
\phi_{\xi}^{*}: C(G)^{*} \rightarrow B^{*}
$$

For $\mu$ right invariant in $C(G)^{*}$ let $\rho=\phi_{\xi}^{*} \mu$. Then

$$
\rho\left(x_{0}\right)=\left(\phi_{\xi}^{*} \mu\right)\left(x_{0}\right)=\mu\left(\phi_{\xi} x_{0}\right) \neq 0
$$

and thus $\rho \neq 0$. Let $S \in G$ and $x \in B$. Then

$$
\rho(S x)=\left(\phi_{\xi}^{*} \mu\right)(S x)=\mu\left(\phi_{\xi}(S x)\right)=\mu\left(r_{s}\left(\phi_{\xi} x\right)\right)=\mu\left(\phi_{\xi} x\right)=\left(\phi_{\xi}^{*} \mu\right)(x)=\rho(x) .
$$

Thus $B$ is $G$-amenable by the element $\rho=\phi_{\xi}^{*} \mu$ in $B^{*}$.

THEOREM 3. Suppose there is an $x_{0}$ in $B$ such that 0 is not in then closed convex hull $\mathrm{L}\left(x_{0}\right)$ of $\left\{S x_{0}: S \in G\right\}$. Then $B$ is $G$-amenable of $G$ is right amenable. Conversely, if $B$ is $G$-amenable then there is an $x_{0}$ in $B$ such that $0 \notin K\left(x_{0}\right)$.

Proof. Let $x_{0} \in B$ be such that $0 \notin K\left(x_{0}\right)$, and $\mu$ a right invariant element of $C(G)^{*}$. Then the Support Theorem in an LCS [1] guarantees the existence of an $\xi \in B^{*}$ such that

$$
\xi(0)<\delta=\inf \left\{\xi(x): x \in K\left(x_{0}\right)\right\} .
$$

It is easily shown that

$$
\left[\phi_{\xi} x_{0}-\left(\frac{1}{2} \delta\right) e\right](S) \geqq 0 \text { for all } S \in G .
$$


Then, since $\mu$ is a right invariant mean in $C(G)^{*}$ we have $\mu\left[\phi_{\xi} x_{0}-\left(\frac{1}{2} \delta\right) e\right] \geqq 0$. Hence

$$
\begin{aligned}
\mu\left(\phi_{\zeta} x_{0}\right) \geqq \mu\left(\frac{1}{2} \delta(e)\right) & =\frac{1}{2} \delta[\mu(e)] \\
& =\frac{1}{2} \delta>0 .
\end{aligned}
$$

By Lemma $3 B$ is $G$-amenable.

Conversely, suppose $B$ is $G$-amenable by $\rho \in B^{*}$. Then $\rho(S x)=\rho(x)$ for all $x \in B$ and $S \in G$. Find $x_{0} \in B$ such that $\rho\left(x_{0}\right)=1$. The convex combinations $\sum_{k=1}^{n} t_{k} S_{k} x_{0}$ are dense in $K\left(x_{0}\right)$. Also

$$
\begin{aligned}
\rho(y)=\rho\left(\sum_{k=1}^{n} t_{k} S_{k} x_{0}\right)=\sum_{k=1}^{n} t_{k} \rho\left(S_{k} x_{0}\right) & =\sum_{k=1}^{n} t_{k} \rho\left(x_{0}\right) \\
& =\sum_{k=1}^{n} t_{k}=1 .
\end{aligned}
$$

Hence $\rho$ is identically one on the (weakly) closed convex hull of $\left\{S x_{0}: S \in G\right\}$ Hence $0 \notin K\left(x_{0}\right)$.

Corollary 2. Suppose $G$ is right amenable and $G$ has a nonzero fixed point $x_{0}$ in $B$. Then $B$ is G-amenable.

We will now impose further restrictions on $G$ to insure the $G$-amenability of $B$.

Definition. The semigroup $G \subseteq \mathscr{L}(B)$ is called almost periodic $(A P)$ if the norm closure of $0(x)=\{S x: S \in G\}$ is compact for each $x \in B$.

Lemma 4. If $G$ is $A P$, then for all $x \in B$ and $\xi \in B^{*}$ the set

$$
c(x)=\left\{r_{s}\left(\phi_{\xi} x\right): S \in G\right\}
$$

is relatively compact in $C(G)$.

Proof. Let $x \in B$ and $\xi \in B^{*}$. To show $c(x)$ is relatively compact in $C(G)$, let $\left\{r_{s_{z}} \phi_{\xi} x\right\}$ be any net in $c(x)$. Since $O(x)$ is relatively compact in $B$, there is a subnet $\left\{S_{n}\right\}$ of $\left\{S_{\alpha}\right\}$ such that $S_{n} x \rightarrow y$ for some $y \in B$. Since $\phi_{\xi}$ is continuous

$$
\phi_{\xi}\left(S_{n} x\right) \rightarrow \phi_{\xi} y \text { in } C(G) \text {. }
$$

Hence

$$
r_{s_{-}}\left(\phi_{\xi} x\right)=\phi_{\xi}\left(S_{n} x\right) \rightarrow \phi_{\xi} y
$$

and $c(x)$ is relatively compact in $C(G)$.

The next theorem is suggested by the proof of Theorem 1 of Chapter XI of [4]. 
Lemma 5. Suppose $G$ is $A P$ and has a nonzero fixed point $x_{0}$. Let $\phi_{\xi}$ be an isometry and $D$ rhe Banach space $\left\{\phi_{\xi} x: x \in B\right\}=\phi_{\xi} B \subset C(G)$. Then

(i) $e \in D$, where $e(S)=1$ for all $S \in G$.

(i) $R_{G}=\left\{r_{s}: S \in G\right\} \subseteq \mathscr{L}(D)$.

(iii) $R_{G}^{*}$ is equicontinuous on the set

$$
K=\left\{\beta \in D^{*}:\|\beta\| \leqq 1, \beta(e)=1 \text { and } \beta(f) \geqq 0 \text { for all } f \geqq 0 \text { in } D\right\}
$$

where the topology on $K$ is the induced $w^{*}$-topology.

Proor. Since $\phi_{\xi}$ is an isometry we have $\left\|\phi_{\xi} x_{0}\right\|=\left\|x_{0}\right\| \neq 0$ and $\phi_{\xi} x_{0} \neq 0$. Also

$$
\left(\phi_{\xi} x_{0}\right)(S)=\xi\left(S x_{0}\right)=\xi\left(x_{0}\right) ;
$$

thus $\phi_{\xi} x_{0}$ is a nonzero constant function and hence $e \in D$.

To show (ii), let $f=\phi_{\xi} x$ be in $D$ and $S \in G$. Then

$$
r_{s} f=r_{s} \phi_{\xi} x=\phi_{\xi}(S x) \in \phi_{\xi} B=D .
$$

Hence (ii) is shown.

To show (iii) let $V$ be any $w^{*}$-open neighborhood of zero in $D^{*}$. Then there are $x_{1}, x_{2}, \cdots, x_{n}$ in $B$ and $\varepsilon>0$ such that

$$
V=\left\{\rho \in D^{*}:\left|\rho\left(\phi_{\xi} x_{i}\right)\right|<\varepsilon, i=1,2, \cdots, n\right\} .
$$

By Lemma 4 we know

$$
M=\bigcup_{k=1}^{n}\left[r_{s} \phi_{\xi} x_{i}: S \in G\right\}
$$

is relatively compact in $C(G)$ and hence $D . M$ is thus totally bounded and there exists $y_{1}, y_{2}, \cdots, y_{m}$ in $B$ such that each $r_{s} \phi_{\xi} x_{i}$ is at a distance of less than $\varepsilon / 4$ from some $\phi_{\xi} x_{i}$. Let

$$
U=\left\{\gamma \in D^{*}:\left|\gamma\left(\phi_{\xi} y_{i}\right)\right|<\frac{1}{2} \varepsilon, i=1,2, \cdots, n\right\} .
$$

Then for $\beta_{1}, \beta_{2} \in K$ and $\beta_{1}-\beta_{2} \in U$ we have for each $S \in G$

$$
\begin{aligned}
\mid\left[r_{s}\left(\beta_{1}-\beta_{2}\right)\left(\phi_{\xi} x_{i}\right) \mid=\right. & \left|\left(\beta_{1}-\beta_{2}\right)\left(r_{s} \phi_{\xi} x_{i}\right)\right| \\
\leqq & \left|\left(\beta_{1}-\beta_{2}\right)\left(r_{s} \phi_{\xi} x_{i}-\phi_{\xi} y_{i}\right)\right| \\
& +\left|\left(\beta_{1}-\beta_{2}\right)\left(\phi_{\xi} y_{i}\right)\right| \\
\leqq & 2|| r_{s} \phi_{\xi} x_{i}-\phi_{\xi} y_{i} \| \\
& +\left|\left(\beta_{1}-\beta_{2}\right)\left(\phi_{\xi} y_{i}\right)\right| \\
< & 2 \frac{1}{4} \varepsilon+\frac{1}{2} \varepsilon=\varepsilon .
\end{aligned}
$$

Thus $r_{s}^{*}\left(\beta_{1}-\beta_{2}\right) \in V$ and (iii) is proved. 
THEOREM 4. If in addition to the hypothesis of Lemma 5 we assume $G$ is a group, then $D$ is $R_{G^{-}}$amenable and $B$ is G-amenable.

Proof. As above let

$$
K=\left\{\beta \in D^{*}:\|\beta\| \leqq 1, \beta(e)=1 \text { and } \beta(f) \geqq 0 \text { for all } f \geqq 0 \text { in } D\right\} .
$$

Clearly $r_{s}^{*}: K \rightarrow K$ for each $S \in G$. Hence $R_{G}^{*}$ is a group of operators equicontinuous on the compact convex set $K$ and $R_{G}^{*}: K \rightarrow K$. Then by Kakutanis'. Theorem [4], $R_{G}^{*}$ has a fixed point $\Theta$. Since $\Theta \in K, \Theta(e)=1$ and $\Theta \neq 0$. For $f \in D$ we have

$$
\Theta\left(r_{s} f\right)=\left(r_{s}^{*} \Theta\right)(f)=\Theta(f)
$$

and $D$ is $R_{G}$-ameneable.

To show $B$ is $G$-amenable let $x \in B$ and $S \in G$. Then

$$
\left(\phi_{\xi}^{*} \Theta\right)(S x)=\Theta\left[\phi_{\xi}(S x)\right\}=\Theta\left[r_{s}\left(\phi_{\xi} x\right)\right]=\Theta\left(\phi_{\xi} x\right)=\left(\phi_{\xi}^{*} \Theta\right)(x)
$$

and hence $\rho=\phi_{\xi}^{*} \Theta$ is a $G$-invariant element of $B^{*}$. Since $\phi_{\xi}$ is an isometry from $B$ onto $D$ and $\Theta \neq 0$ we have that

is $1-1$ and $\phi_{\xi}^{*} \Theta \neq 0$.

$$
\phi_{\xi}: D^{*} \rightarrow B^{*}
$$

Corollary 3. Let $G$ be such that (i) there is an $x_{0}$ in $B$ such that $0 \notin K\left(x_{0}\right)$ and (ii) $G^{*}=\left\{S^{*}: S \in G\right\}$ is equicontinuous on the $w^{*}$-closed convex hull of $\left\{S^{*} \beta: S \in G\right\}$ where $\beta$ is such that $\beta\left(S x_{0}\right) \geqq m>0$ for all $S \in G$. Then $B$ is G-amenable.

Proof. Same as that of Theorem 4. Note that the existence of a $\beta \in B^{*}$ such that $\beta\left(S x_{0}\right) \geqq m>0$ for all $S$ is guaranteed by (i) and the Support Theorem.

Another result concerning $G$-amenability is

THEOREM 5. If $G$ has a nonzero fixed point, then $B^{*}$ is $G^{*}$-amenable. Conversely, if $B^{*}$ is $G^{*}$-amenable and $B$ is reflexive, then $G$ has a nonzero fixed point.

Proof. Let $x_{0} \neq 0$ be a fixed point of $G$ and $Q$ the natural map of $B$ into $B^{* *}$; then $Q x_{0} \neq 0$. We have

$$
\left(Q x_{0}\right)\left(S^{*} \beta\right)=\left(S^{*} \beta\right)\left(x_{0}\right)=\beta\left(S x_{0}\right)=\beta\left(x_{0}\right)=\left(Q x_{0}\right)(\beta)
$$

for all $S^{*} \in G^{*}$ and $\beta \in B^{*}$. Hence $B^{*}$ is $G^{*}$-amenable.

If $B^{*}$ is $G^{*}$-amenable by $f \in B^{* *}$ and $B$ is reflexive, then $f=Q x_{0}$ for some $x_{0} \in B$. Let $S \in G$ and $\xi \in B^{*}$. Then

$$
\begin{aligned}
\xi\left(S x_{0}\right)=\left(S^{*} \xi\right)\left(x_{0}\right)=\left(Q x_{0}\right)\left(S^{*} \xi\right) & =f^{*}(S \xi)=f(\xi) \\
= & \left(Q x_{0}\right)(\xi)=\xi\left(x_{0}\right) .
\end{aligned}
$$


Thus $\xi\left(S x_{0}\right)=\xi\left(x_{0}\right)$ for all $\xi \in B^{*}$ and $S \in G$. Since $B^{*}$ is total over $B$ it must be that $S x_{0}=x_{0}$ for all $S \in G$.

There are many elementary geometric conditions one may put on $G$ to insure the $G$-amenability of $B$. Among them is

THEOREM 6. There is a unique G-invariant element $\mu$ of $B^{*}$ if and only if the factor space $L$ has deficiency one where $L$ is the closed subspace generated by

$$
\{S x-x: S \in G, x \in B\} .
$$

Note. By the uniqueness of $\mu$ we mean that if $\Theta$ is also $G$-invariant, then $\beta=t \Theta$ for some real number $t \neq 0$. The proof of this theorem is easy and omitted.

We conclude with a corollary to Corollary 2 .

THEOREM 7. If $G$ has a nonzero fixed point and if $G$ is either a finite group or an abelian semigroup, then $B$ is G-amenable.

Proof. In either case, $G$ is amenable by [2].

\section{References}

[1] M. M. Day, Normed Linear Spaces (Academic Press, 1962).

[2] M. M. Day, 'Amenable Semigroups, Ill.' Jnl. of Math. 1, 1957.

[3] M. M. Day, Semigroups and Amenability, pp. 5-53 of Semigroups (Ed. by K. W. Folley), (Academic Press, New York, 1969).

[4] N. Dunford and J. T. Schwartz, Linear Operarors, Parts I and II, (Interscience, 1958).

[5] F. P. Greenleaf, Invariant Means on Topological Groups, (Van Nostrand, 1969).

[6] E. Hewitt and K. Ross, Abstract Harmonic Analysis, vol. 1, (Academic Press, New York, 1963).

[7] C. R. Rao ( = R. R. Chivukula), 'Invariant Means on Spaces of Continuous or Measurable Functions', Trans. Amer. Math. Soc. 114, (1965), pp. 187-196.

[8] R. N. Rich, 'Simple and Weakly Almost Periodic Transformation Groups', Trans. Amer. Math. Soc. 123, 1966.

[9] C. O. Wilde and T. Jayachandrau, 'Amenable Transformations Semigroups', Journal of the Australian Math. Soc. 12 (1971), 502-510.

Virginia Polytechnic Institute

and State University

Blacksubrg, Virginia

U.S.A.

University of Nebraska

Lincoln, Nebraska

U.S.A. 